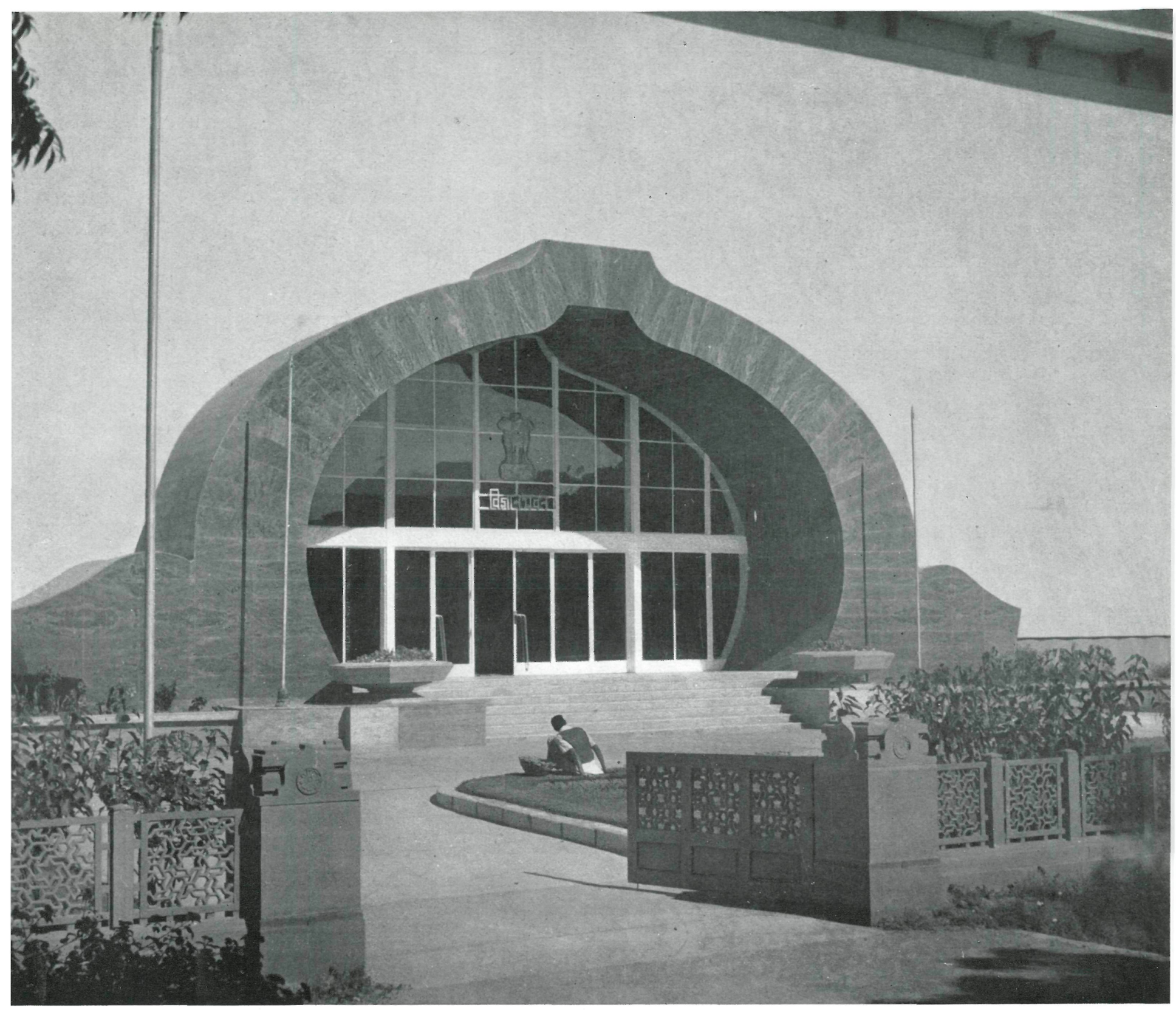

\title{
centro de conferencias en Mueva Delhi
}

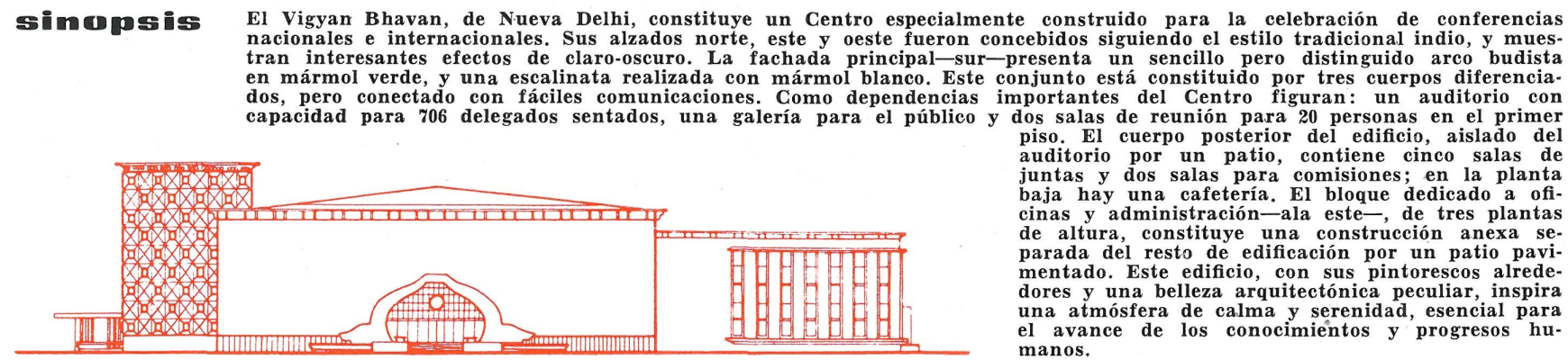



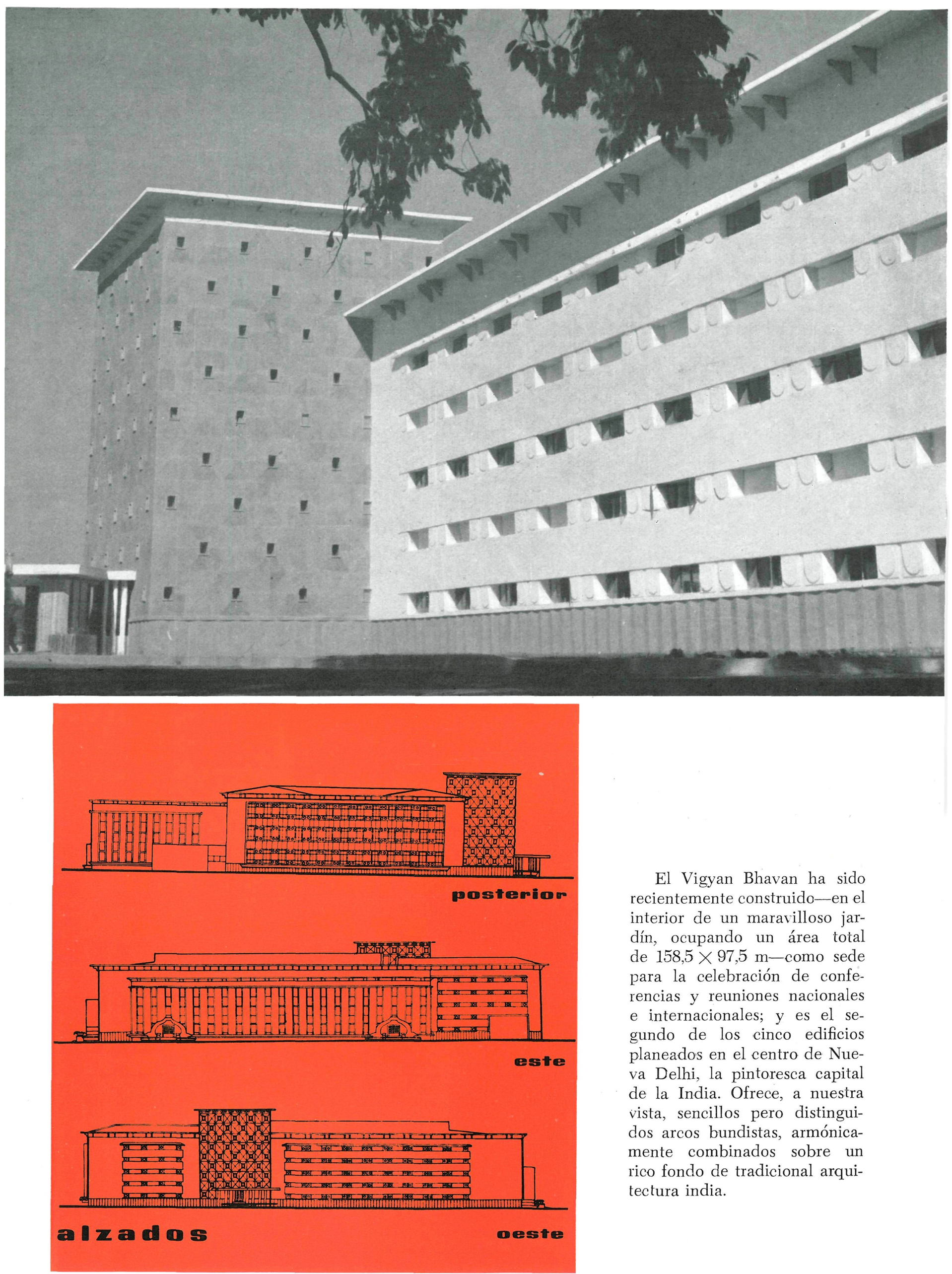

El Vigyan Bhavan ha sido recientemente construido- -en el interior de un maravilloso jardín, ocupando un área total de $158,5 \times 97,5 \mathrm{~m}-$ como sede para la celebración de conferencias y reuniones nacionales e internacionales; y es el segundo de los cinco edificios planeados en el centro de Nueva Delhi, la pintoresca capital de la India. Ofrece, a nuestra vista, sencillos pero distinguidos arcos bundistas, armónicamente combinados sobre un rico fondo de tradicional arquitectura india. 

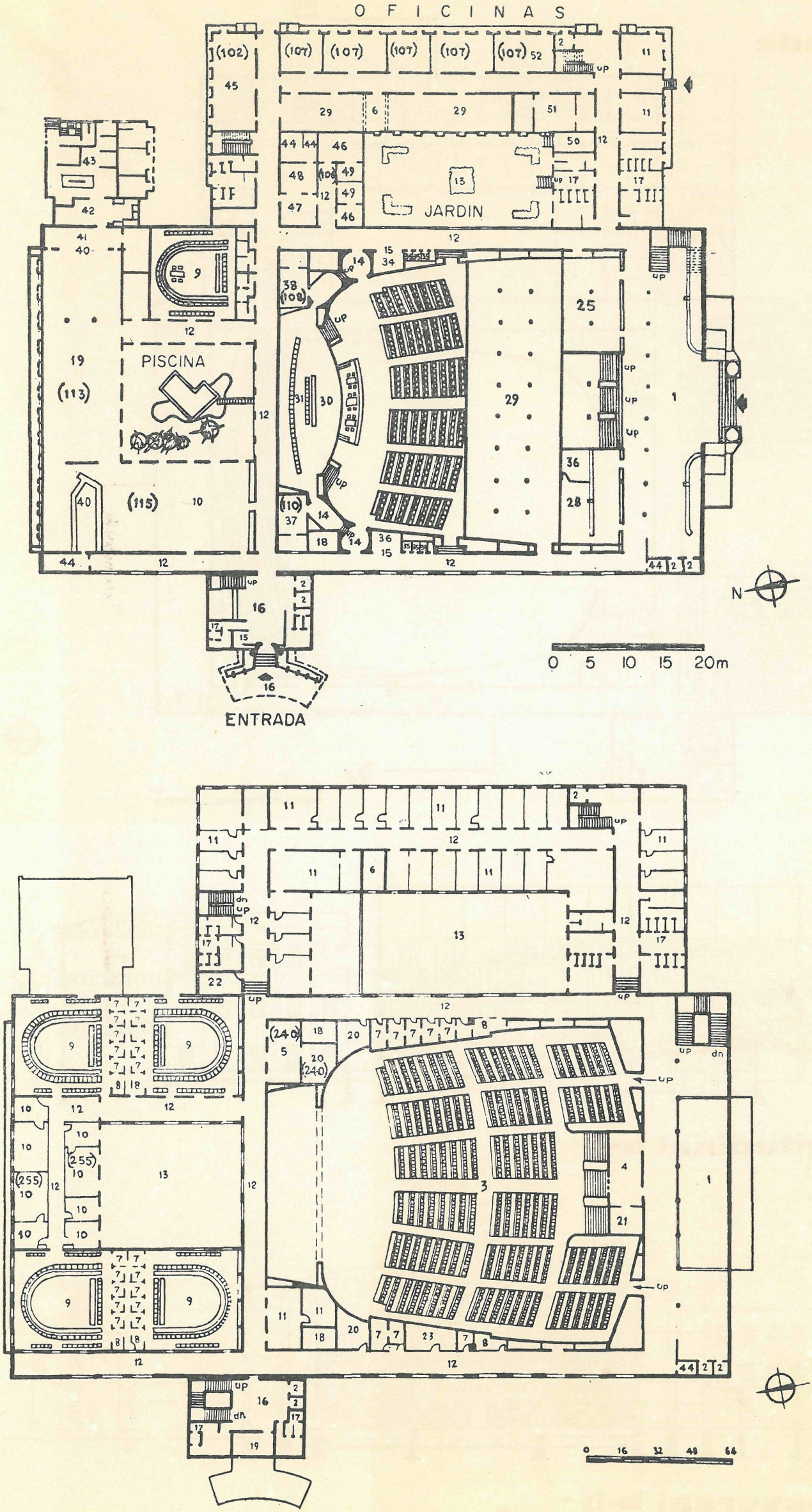

pllamtas

เo

1. Hall. - 2. Ascensores.. - 3 . Reuniones generales.-4. Sala de proyecciones. $\mathbf{- 5}$. Salas de coloquios. - 6. Conductos de aire acondicionado.-7. Cabinas de intérpretes. -8 . Control. - 9. Salas de Juntas. 10. Vestíbulo principal. -11 . Oficinas.-12. Corredores.-13. Patio.-14. Vestíbulo.-15. Mostrador.-16. Entrada reservada a personalidades. -17 . Aseos.-18. Conductos de aire. 19. Prensa. - 20. Habitación disponible. - 21. Almacén de películas. -22 . Instrumentos eléctricos.-23. Galería de fotógrafos.-24. Balcón.-25. Habitación para comisiones. 26. Galería-27. Médico-28. Oficina de turismo y banco.29. Instalación de aire acondicionado-30 Escenario.-31. Presidente. -32 . Orador. -33 . Secretarios, foso de orquesta. 34. Oficina de viajes y transportes. -35 . Teléfonos.-36. Correos.-37. Habitación particular del presidente.-38. Habitación de consejeros.-39. Cafetería.40. Mostrador de servicio.-41. Vestibulo de servicio.-42. Despensa.-43. Cocina.-44. Almacén.-45. Subestación eléctrica.-46. Sección de estudio.-47. Control.-48. Doblaje. -49 . Sonido. -50 . Bombas. -51 . Centralita de teléfonos.-52. Servicio de comunicación a ultramar.

Nota: Las cifras encerradas con paréntesis indican el número de las habitaciones. 

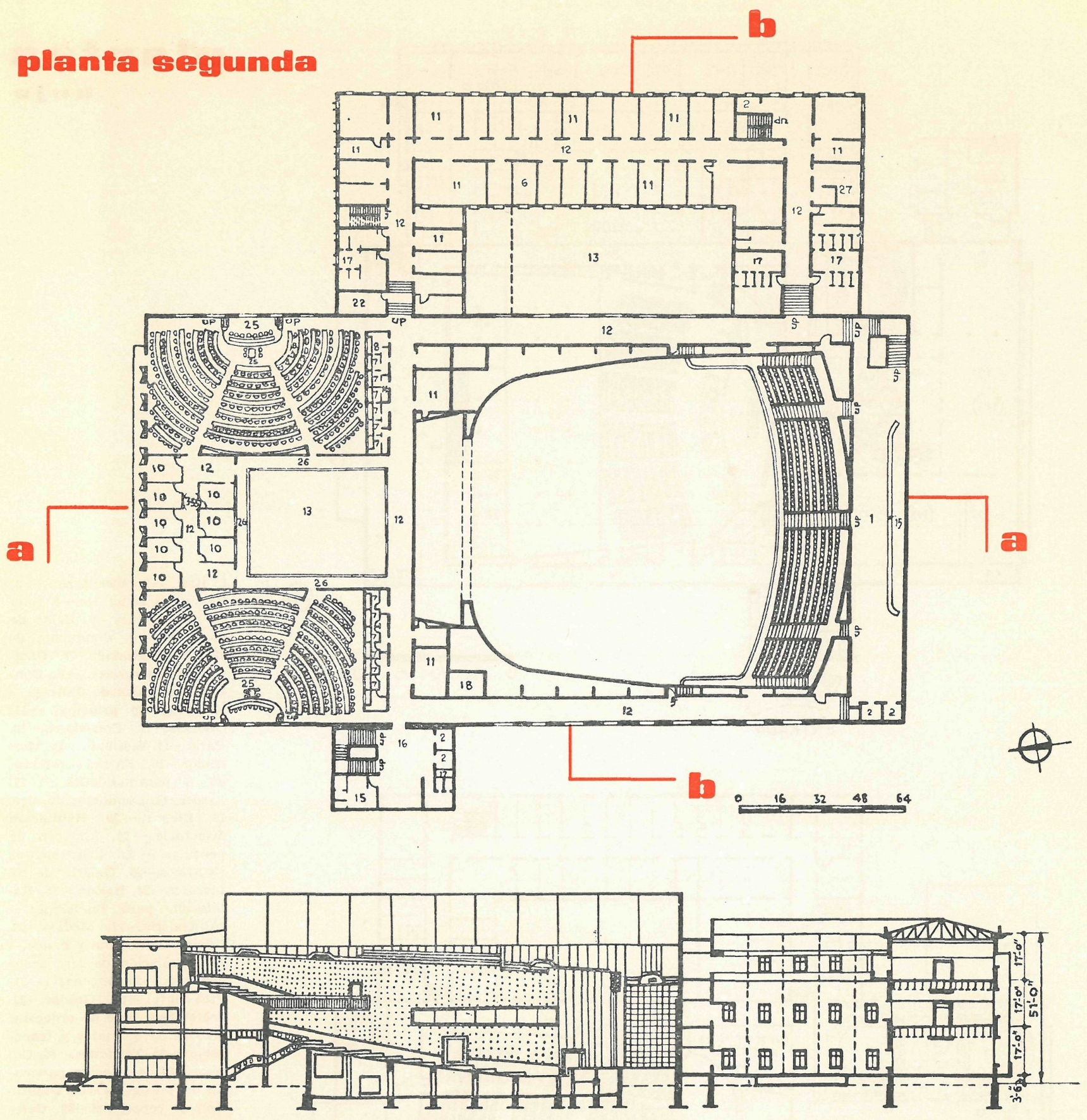

scceión longitudimal a-a

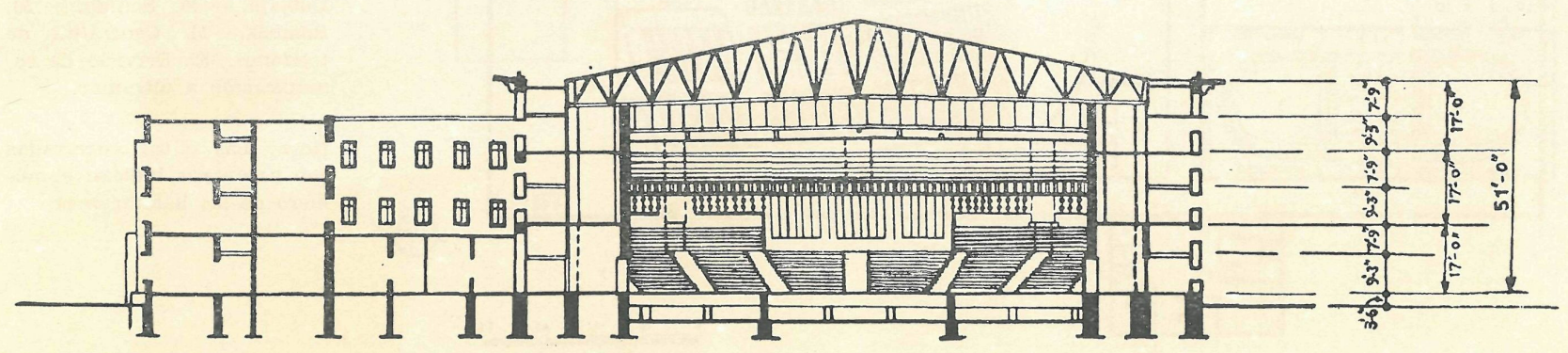

sección treansucresa|b-b 
Los arcos están realizados a base de mármol verde oscuro, sobrepuestos a las lisas fachadas de arenisca. De ellos, el central-en la entrada principal-tiene $7 \mathrm{~m}$ de alto, $11,2 \mathrm{~m}$ de ancho y $2,7 \mathrm{~m}$ de profundidad; los otros son más pequeños y están situados en la fachada oeste.

En cuanto a la arquitectura interior, el cuerpo principal constituye, en realidad, una integración perfecta de pinturas, esculturas y murales. De éstos señalamos el existente en el gran vestíbulo, que describe la vida rural en la India y cubre la parte superior de la abertura arqueada. Otros dos grandes paneles murales decoran la pared sur del vestíbulo en planta baja.

Todo el edificio se eleva sobre un pedestal revestido de arenisca roja.

Una airosa cornisa, sustentada sobre pilares de estilo indio, chapados con arenisca blanca, corona la edificación.

Los alzados norte, este y oeste, asimismo concebidos en el estilo tradicional del país, exhiben numerosos matices e interesantes efectos de claro-oscuro.

El bloque de oficinas y la fachada sur presentan jardineras corridas de hormigón, cuajadas de fragantes y multicolores flores. A la entrada principal se accede por una escalinata de mármol blanco. Materiales de tan distinta textura-chapado de arenisca roja, pilares de arenisca blanca, super- 
ficies estucadas...-, proporcionan a tste conjunto una deliciosa entonación de colores, calidad y riqueza.

Por lo que respecta a su organización interior podemos distinguir tres cuerpos independientes, pero conectados de forma que entre los mismos exista fácil comunicación.

Como dependencias importantes de este Centro figuran: el auditorio, con capacidad para 706 delegados sentados; una galería pública, con cabida para 322 asistentes; y dos salas de reunión, para 20 personas, en el primer piso, cerca del escenario del auditorio.

El cuerpo posterior del edificio se halla separado del salón de actos por un patio-con un pequeño estanque en el centro-y alberga cinco salas de Juntas, cada una capaz para 50 delegados y 20 observadores; dos salas para comisiones, con 255 asientos.

En planta baja existe una cafetería que puede servir, al mismo tiempo, a 300 personas; y dos salones de descanso en el primero y segundo pisos.

La agrupación de entradas y salas de reunión ha sido cuidadosamente estudiada, con el fin de asegurar una cómoda circulación entre ellas y, además, están situadas en forma tal que los delegados pueden ir de un sitio a otro sin ser interrumpidos por los representantes de la prensa, el público o los administrativos. 
soula de conferencius secum doura
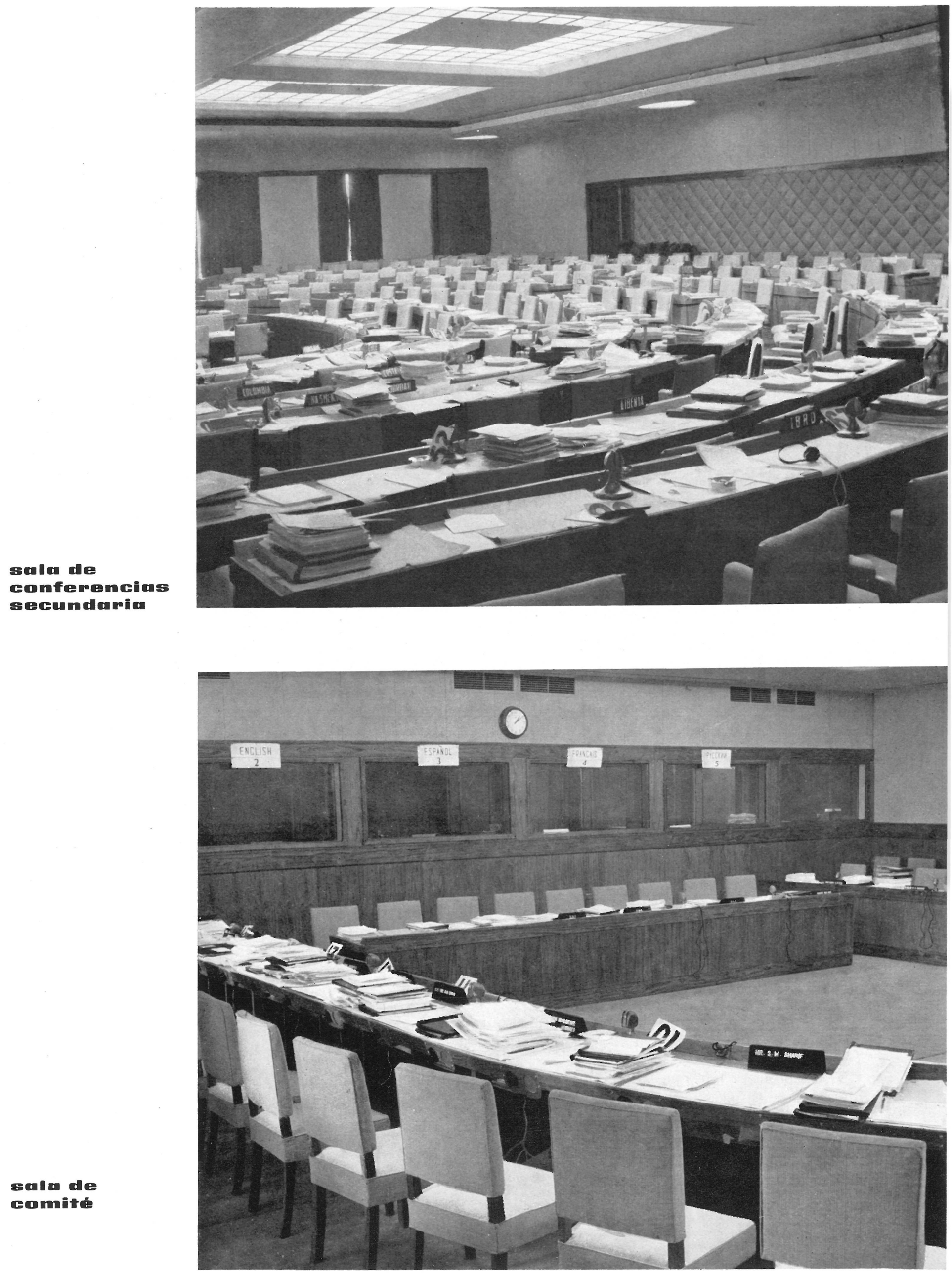

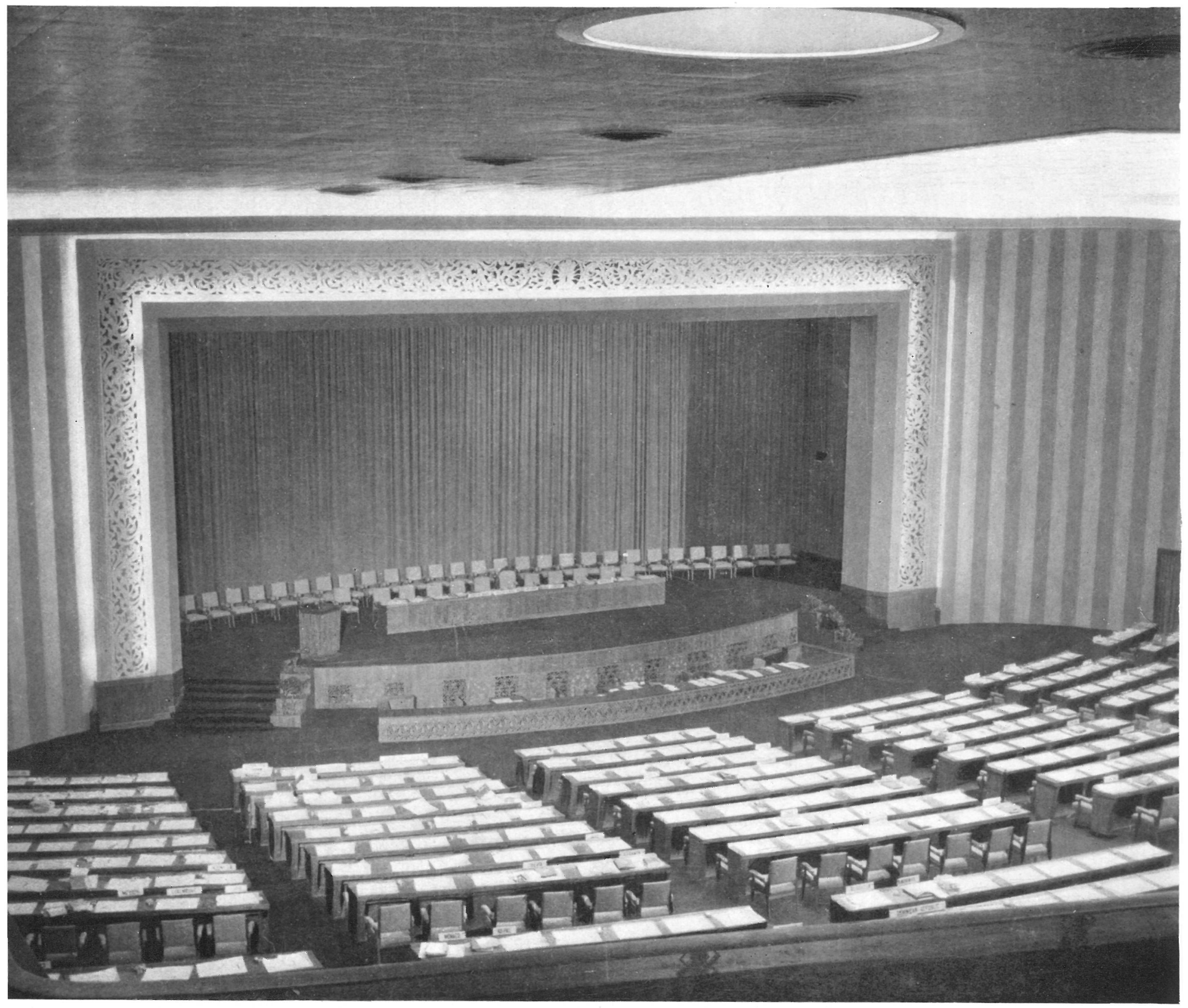

La sala destinada a la prensa está emplazada cerca de la entrada, en conexión con el vestíbulo, próxima al auditorio, con objeto de poder ser utilizada por los delegados que deseen un rápido y directo contacto con los representantes de la prensa.

Al mismo tiempo, la circulación del público queda desviada por medio de un ascensor especial que conduce desde el vestíbulo a la galería destinada a visitantes.

Más allá, el bloque que alberga las oficinas y la administración, de tres plantas de altura, constituye una construcción anexa y está separada de los restantes por un patio pavimentado.

El Vigyan Bhavan, con sus pintorescos alrededores y una belleza arquitectónica que le es peculiar, inspira una atmósfera de calma y serenidad tan esencial para el avance de los conocimientos y progresos humanos.

S. SARWAL, ingeniero. 


\section{résumé̉ S summmary}

\section{Centre de conféremcess di New Delli}

S. Sarwal, ingénieur.

Le Vigyan Bhavan, de New Delhi, est un centre spécialement construit pour la célébration de conférences nationales et internationales.

Ses façades nord, est et ouest ont été conçues selon le style traditionnel indien et montrent d'intéressants contrastes de clair-obscur. La facade principale, au sud, présente un arc bouddhiste en marbre vert, simple mais distingué, et un perron réalisé en marbre blanc.

Cet ensemble est composé de trois corps différents, mais unis par de faciles communications.

Comme dépendances importantes du «Centre» figurent: un auditorium capable d'abriter 706 délégués assis, une galerie pour le public et deux salles de réunion pour vingt personnes au premier étage.

Le corps postérieur de l'édifice, isolé de l'auditorium par une cour, compte cinq salles de réunion et deux salles pour commissions. Le rez-de-chaussée abrite une cafeteria.

Le bloc destiné aux bureaux et à l'administration-aile est-à trois étages, constitue une construction annexe séparée des autres édifices par une cour dallée.

Cet ensemble, avec ses alentours pittoresques et sa beauté architectonique caractéristique, crée une atmosphère de calme et de sérénité, essentielle pour l'épanouissement des connaissances et du progrès humains.

\section{Comgress cemtre at New Dellhi}

S. Sarwal, engineer.

Vigyan Bhavan, in New Delhi, is a centre specially built to hold in it national and international meetings and congresses.

The north, east and west faces of the building follow closely the traditional local Indian style. The main frontage, to the south, has a simple but fine Buddhist arch in green marble, and wide steps leading to it in white marble. This centre consists of three distinct, but well integrated, buildings.

The main facilities of this project are: an auditorium with seating accommodation for 706 delegates, a public gallery, and two meeting rooms for 20 people each, on the first floor.

A separate section houses 5 committee rooms, two rooms for other group meetings, and a cafeteria, on the lower floor.

The offices and other administrative services have been placed in the east wing, which is three storeys high, and is separated from the rest of the centre by a paved patio.

The peculiar beauty of this project and the surrounding landscape's picturesque charm induce a sense of relaxation that is indeed conducive to the better success of international meetings.

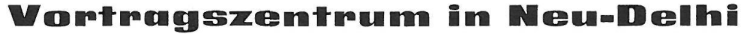

s. Sarwal, Bauingenieur.

Vigyan Bhavan in Neu-Delhi bildet ein besonders für die Abhaltung von nationalen und internationalen Vorträgen erbautes Zentrum.

Seine Aufrisse gegen Norden, Osten und Westen wurden so gestaltet, dass sie dem herkömmlichen indischen Stil folgen; sie zeigen interessante Wechselwirkungen zwischen hell und dunkel. Die Hauptfassade gegen Süden weist einen einfachen, aber vornehmen buddhistischen Bogen aus grünem Marmor und eine in weissem Marmor ausgeführte Freitreppe auf.

Diese Gesamtheit besteht aus drei unterschiedenen Gehäusen, die jedoch durch leichte Verbindungen zusammenhängen.

Als bedeutende Nebengebäude des «Zentrums» spielen eine Rolle: ein Hörsaal mit Sitzplätzen für y06 Abgeordnete, eine Galerie für das Publikum und zwei Versammlungssäle für 20 Personen im ersten Geschoss.

Der rückwärtige Teil des Gebäudes, durch einen Hof vom Hörsaale getrennt, enthält 5 Säle für Sitzungen und 2 Säle für Abordnungen; im Erdgeschoss befindet sich ein Kaffee.

Der für Bureau- und Verwaltungsräume bestimmte Block-der Ostflügel-mit drei Stockwerken bildet einen vom übrigen Gebäude durch einen gepflasterten Hof getrennten Anbau.

Dieses Gebäude mit seiner malerischen Umgebung und einer einzigartigen architektonischen Schönheit ruft eine Atmosphäre der Ruhe und Gelassenheit hervor, welche für das Voranschreiten der menschlichen Kenntnisse und Fortschritte wesentlich ist. 